\title{
Long-acting insulin analogs exposure and cancer specific mortality in patients with diabetes mellitus
}

Sorin loacara ${ }^{1,2}$, Cristian Guja ${ }^{1}$, Constantin lonescu-Tirgoviste ${ }^{1}$, Simona Fica², Sorina Martin², Michael Roden ${ }^{3}$

1 "I. Pavel" Outpatient Clinic, Bucharest, Romania, 2 "Elias" Hospital, Bucharest, Romania

${ }^{3}$ Institute for Clinical Diabetology, German Diabetes Center, Düsseldorf, Germany

\section{AIM}

To test the hypothesis that exposure to insulin glargine might be associated with increased risk of cancer mortality compared with human basal insulin preparations.

\section{MATERIALS AND METHODS}

All consecutive diabetes patients aged over 40 years, residing in a major urban area were screened at their first diabetes outpatient visit between 01/01/2001-12/31/2008 $(n=79869)$. Exclusion criteria were insulin treatment at screening $(\mathrm{n}=14752)$, no insulin treatment until $12 / 31 / 2008(n=55795),<6$ months of glucose-lowering treatment alone before insulin initiation $(n=1154)$, insulin prescription before glargine became available $(04 / 17 / 2003, n=1761)$, age $<40$ or $\geq 80$ years at first insulin prescription $(n=406),<6$ months of insulin treatment following insulin initiation $(n=1011)$. A total 4990 subjects were followed-up for death based on death certificate, until 12/31/2011, using data from National Institute of Statistics.

\section{RESULTS II}

Figure 1. Cancer deaths cumulative incidence functions. Green line, females exposed to glargine; maroon line, females unexposed to glargine; blue lines, males exposed to glargine; red line, males unexposed to glargine.

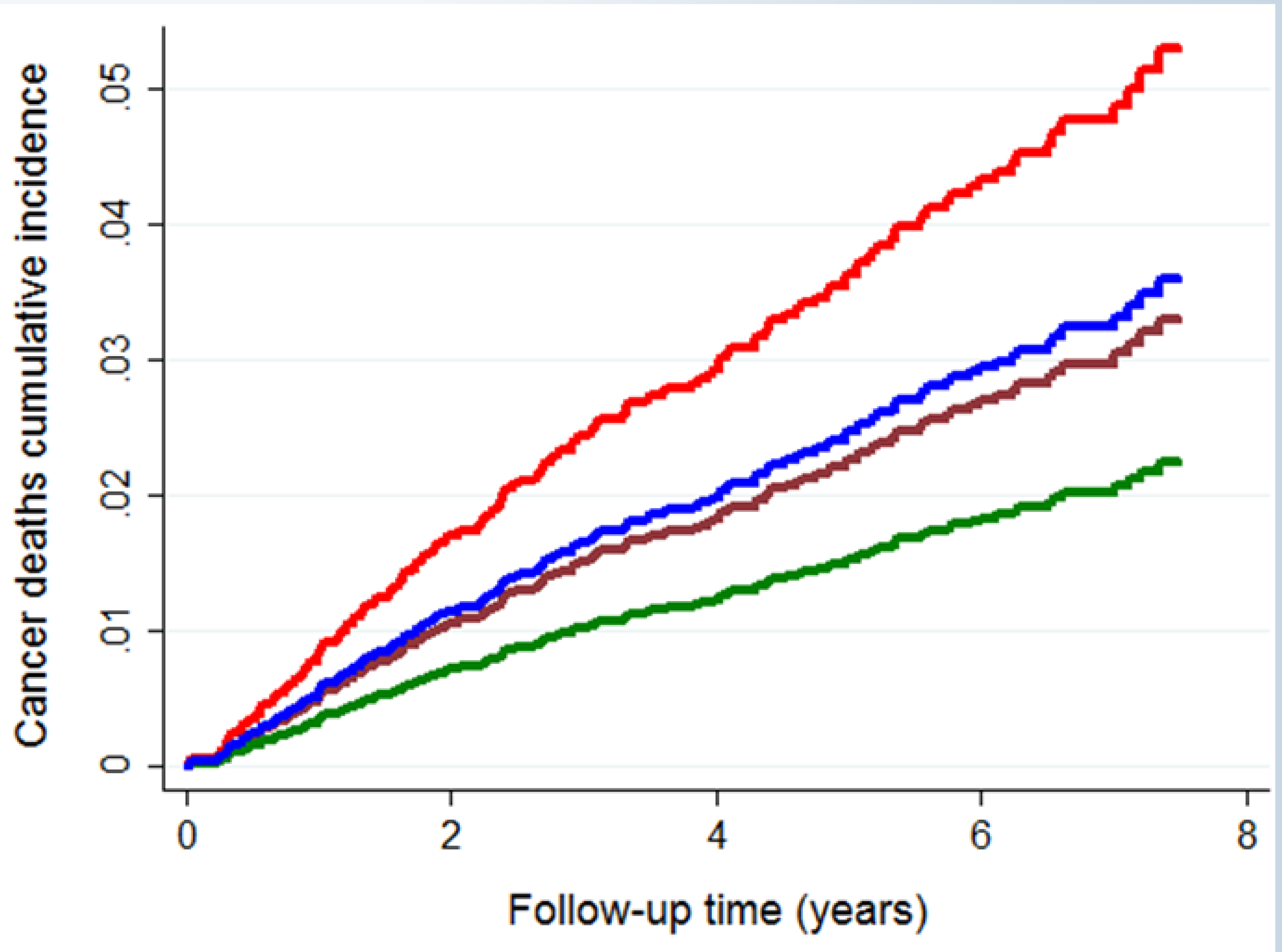

\section{RESULTS I}

Mean baseline age was $62 \pm 9$ years, and follow-up $4.7 \pm 1.9$ years. Glargine cumulative dose exposure significantly lowered cancer mortality risk, subhazard ratio (SHR) 0.94 (95\% CI 0.89-0.99, p=0.033).

Table 1. Competing risk analysis of site-specific cancer mortality

\begin{tabular}{lcc}
\hline Cancer site & $\begin{array}{c}\text { Cumulative exposure } \\
\text { SHR }(\mathrm{Cl} 95 \%)\end{array}$ & $\begin{array}{c}\text { Cumulative dose } \\
\text { SHR }(\mathrm{Cl} 95 \%)\end{array}$ \\
\hline Lung $(\mathrm{n}=36)$ & $1.008(0.965-1.054)^{\mathrm{NS}}$ & $0.964(0.897-1.036)^{\mathrm{NS}}$ \\
Colorectal $(\mathrm{n}=20)$ & $0.887(0.657-1.197)^{\mathrm{NS}}$ & $0.913(0.729-1.144)^{\mathrm{NS}}$ \\
Female genital $(\mathrm{n}=18)$ & $1.022(0.895-1.167)^{\mathrm{NS}}$ & $0.956(0.823-1.110)^{\mathrm{NS}}$ \\
Liver $(\mathrm{n}=15)$ & $0.985(0.906-1.072)^{\mathrm{NS}}$ & $1.022(0.950-1.099)^{\mathrm{NS}}$ \\
Pancreatic $(\mathrm{n}=15)$ & $5^{*} 10^{-6}\left(6^{*} 10^{-9}-0.005\right)^{* *}$ & $9^{*} 10^{-5}\left(1 * 10^{-7}-0.058\right)^{* *}$ \\
Breast $(\mathrm{n}=13)$ & $0.762(0.663-0.877)^{* *}$ & $0.849(0.769-0.937)^{* *}$ \\
Urinary tract $(\mathrm{n}=11)$ & $0.885(0.761-1.030)^{\mathrm{NS}}$ & $0.933(0.828-1.051)^{\mathrm{NS}}$ \\
\hline & & \\
NS not significant, ${ }^{*} \mathrm{p}<0.05,{ }^{* *} \mathrm{p}<0.01$ &
\end{tabular}

\section{CONCLUSIONS}

The cumulative dose exposure to insulin glargine was associated with a lower risk of cancer mortality in general, and of breast and pancreatic cancer in particular.

- No glargine associated "harm" was found even after additional "fixed" cohort or propensity score analyses were performed.

\section{CONTACT INFORMATION}

Dr. Sorin Ioacara

"Elias" Hospital, Bucharest, Romania.

E-mail: drsorin@yahoo.com 